\title{
NA48: Results on Rare Decays and Future Prospects
}

\author{
E. Mazzucato ${ }^{\mathrm{a}}$ * \\ ${ }^{a}$ CEA Saclay, DAPNIA/SPP \\ 91191 Gif-sur-Yvette Cedex, France
}

Recent results obtained by the NA48 Collaboration on rare neutral kaon and hyperon decays are presented. These include measurements performed with the simultaneous $K_{L}$ and $K_{S}$ beams during the $\varepsilon^{\prime} / \varepsilon$ programme as well as investigations of rare $K_{S}$ and neutral hyperon decays using high intensity proton beams at the $K_{S}$ target station. Future projects aiming to search for very rare $K_{S}$ decays and to study direct $\mathrm{CP}$ violation with charged kaon beams are also discussed.

\section{INTRODUCTION}

The NA48 experiment at CERN has been designed to measure the direct $C P$ violation parameter $\operatorname{Re}\left(\varepsilon^{\prime} / \varepsilon\right)$ with a precision of about $2 \times 10^{-4}$. The quality of the NA48 simultaneous $K_{L}$ and $K_{S}$ beams [1] together with its high resolution detectors and its high capability trigger and data acquisition systems [2] allow to perform concurrently a thorough investigation of rare neutral kaon decays. In particular, the use of intense proton beams at the $K_{S}$ target station offers the possibility to search for very rare modes like $K_{S} \rightarrow \pi^{0} e^{+} e^{-}$or $K_{S} \rightarrow \pi^{0} \pi^{0} \pi^{0}$ and to study neutral hyperon decays with high sensitivities.

In the first part of my talk, I briefly review recent results on rare kaon decays obtained by NA48 during the 1997-1999 period devoted primarily to direct CP violation studies in $K^{0} \rightarrow 2 \pi$. Then, I discuss several measurements performed in 1999 and in 2000 with a dedicated high intensity $K_{S}$ beam. Finally, I present two proposals submitted by the NA48 Collaboration as possible extensions to the present physics programme beyond 2001.

*On behalf of the NA48 Collaboration: Cagliari, Cambridge, CERN, Dubna, Edinburgh, Ferrara, Firenze, Mainz, Orsay, Perugia, Pisa, Saclay, Siegen, Torino, Vienna, Warsaw.

\section{RARE DECAYS WITH $K_{L}$ AND $K_{S}$ BEAMS}

During the 1997-1999 period, the typical $K_{L}$ and $K_{S}$ fluxes available for the $\operatorname{Re}\left(\varepsilon^{\prime} / \varepsilon\right)$ measurement were respectively $2 \times 10^{7}$ and $3 \times 10^{2}$ per SPS spill at the entrance of the fiducial decay volume of the experiment. The corresponding Single-Event-Sensitivity (SES) in one year of data taking are about $3 \times 10^{-10}$ for $K_{L}$ decays and about $1.5 \times 10^{-7}$ for $K_{S}$ decays, assuming an overall acceptance of $10 \%$ after all analysis cuts. As we will see below, the SES value for $K_{S}$ decays can be significantly improved by using proton beams at the $K_{S}$ target station with an intensity several hundred times higher.

A wide range of rare $K_{L}$ decay modes have been investigated from 1997 until 1999 during the $\varepsilon^{\prime} / \varepsilon$ run. Their study aims at probing chiral dynamics in non-leptonic decays, at the understanding of the weak $K_{L} \rightarrow \gamma \gamma^{*}$ and $K_{L} \rightarrow \gamma^{*} \gamma^{*}$ transitions, $\mathrm{CP}$ violation, etc.

I concentrate here on few topics for which very recent results have been obtained by the NA48 Collaboration.

2.1. $K_{L} \rightarrow \pi^{+} \pi^{-} e^{+} e^{-}$

The investigation of the $K_{L} \rightarrow \pi^{+} \pi^{-} e^{+} e^{-}$decay mode provides a novel way to probe $\mathrm{CP}$ violation in the neutral kaon system. The amplitude for this decay is dominated by two components: one from the $\mathrm{CP}$-conserving direct emission process associated with a magnetic dipole 
transition (M1), the other one from the CPviolating $K_{L} \rightarrow \pi^{+} \pi^{-} e^{+} e^{-}$decay with inner bremsstrahlung. The interference of the $\mathrm{CP}$-even and CP-odd amplitudes produces a CP-violating circular polarization of the virtual photon which gives rise to a large asymmetry in the distribution of the angle $\phi$ between the normals to the $\pi^{+} \pi^{-}$ and $e^{+} e^{-}$planes in the kaon centre-of-mass system $[3,4]$ :

$\frac{d \Gamma}{d \phi}=\Gamma_{1} \cos ^{2} \phi+\Gamma_{2} \sin ^{2} \phi+\Gamma_{3} \sin \phi \cos \phi$

A non-zero value of $\Gamma_{3}$ constitues an unambiguous signature of $\mathrm{CP}$ violation which can be seen in the differential decay rate $d \Gamma / d \phi$.

During the 1998 and 1999 run periods, the NA48 experiment collected more than 1300 good events using a dedicated four-track trigger. The identification of the $K_{L} \rightarrow \pi^{+} \pi^{-} e^{+} e^{-}$events relies on the very good tracking performance of the magnetic spectrometer and on the $e / \pi$ separation obtained with the high resolution LKr electromagnetic calorimeter. The main source of background is due to $K_{L} \rightarrow \pi^{+} \pi^{-} \pi_{D}^{0}$ where $\pi_{D}^{0}$ denotes here the Dalitz decay $\pi_{D}^{0} \rightarrow e^{+} e^{-} \gamma$. This background contribution is more than three orders of magnitude larger than the signal. It can be suppressed by the use of the well-known $P_{0}^{\prime 2}$ kinematic variable:

$$
\begin{aligned}
P_{0}^{\prime 2}= & \frac{1}{4\left(M_{\pi \pi}^{2}+\left(P_{\perp}^{2}\right)_{\pi \pi}\right)}\left\{\left(M_{K}^{2}-M_{\pi^{0}}^{2}-M_{\pi \pi}^{2}\right)^{2}\right. \\
& \left.-4 M_{\pi^{0}}^{2} M_{\pi \pi}^{2}-4\left(P_{\perp}^{2}\right)_{\pi \pi} M_{K}^{2}\right\} .
\end{aligned}
$$

$P_{0}^{\prime 2}$ is greater than zero for $K_{L} \rightarrow \pi^{+} \pi^{-} \pi_{D}^{0}$ decays while it is mostly negative for $K_{L} \rightarrow$ $\pi^{+} \pi^{-} e^{+} e^{-}$events.

Photons from $K_{L} \rightarrow \pi^{+} \pi^{-} \gamma$ decays which are converted in the kevlar window located at the end of the fiducial kaon decay region can be eliminated by requiring a $2 \mathrm{~cm}$ separation between electron tracks in the first drift chamber. Figure 1 shows the invariant mass $M_{\pi \pi e e}$ distribution for $K_{L} \rightarrow \pi^{+} \pi^{-} e^{+} e^{-}$candidates after all other analysis cuts have been applied, together with the different background contributions.

The branching ratio of $K_{L} \rightarrow \pi^{+} \pi^{-} e^{+} e^{-}$ is determined using fully reconstructed $K_{L} \rightarrow$

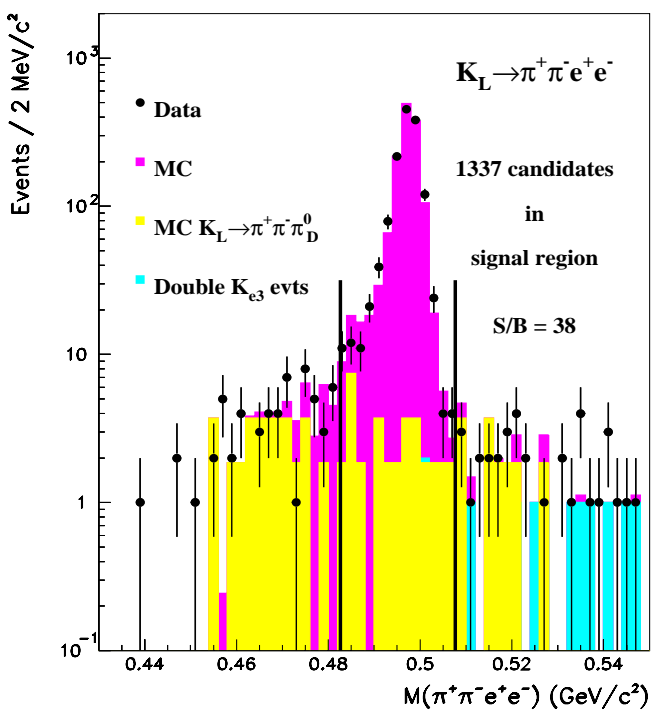

Figure 1. Invariant $\pi^{+} \pi^{-} e^{+} e^{-}$mass distribution for $K_{L} \rightarrow \pi^{+} \pi^{-} e^{+} e^{-}$candidates. The signal region around the kaon mass value is indicated by solid lines.

$\pi^{+} \pi^{-} \pi_{D}^{0} \rightarrow \pi^{+} \pi^{-} e^{+} e^{-} \gamma$ decays as normalization channel. This choice has the advantage that trigger and reconstruction inefficiencies cancel to first order. The acceptance correction for the $K_{L} \rightarrow \pi^{+} \pi^{-} e^{+} e^{-}$process is obtained from a Monte Carlo simulation based on the model of Heiliger and Sehgal [4] with the additional inclusion of a form factor in the M1 direct emission term that takes into account vector meson intermediate states $[5,6]$ :

$F=\tilde{g}_{M 1}\left[1+\frac{a_{1} / a_{2}}{\left(M_{\rho}^{2}-M_{K}^{2}\right)+2 M_{K} E_{\gamma}^{*}}\right]$

Using the values $\tilde{g}_{M 1}=1.35_{-0.17}^{+0.20}$ and $a_{1} / a_{2}=$ $-0.720 \pm 0.029 \mathrm{GeV}^{2} / c^{2}$ measured by $\mathrm{KTeV}[6]$, the NA48 Collaboration obtains the preliminary result: $B R\left(K_{L} \rightarrow \pi^{+} \pi^{-} e^{+} e^{-}\right)=(3.1 \pm 0.1 \pm$ $0.2) \times 10^{-7}$ in fair agreement with the KTeV preliminary branching ratio determination of (3.63土 $0.11 \pm 0.14) \times 10^{-7}[7]$. The asymmetry $\mathcal{A}_{L}$ in the $\sin \phi \cos \phi$ distribution of events, after cor- 
recting for the detector acceptance, is found to be $\mathcal{A}_{L}=(13.9 \pm 2.7 \pm 2.0) \%$ (preliminary), in agreement with theoretical predictions [3,4] and the recently published value of $\mathrm{KTeV}$ [6]. Figure 2 shows the distribution of $K_{L} \rightarrow \pi^{+} \pi^{-} e^{+} e^{-}$ events as a function of $\sin \phi \cos \phi$ before and after acceptance correction.
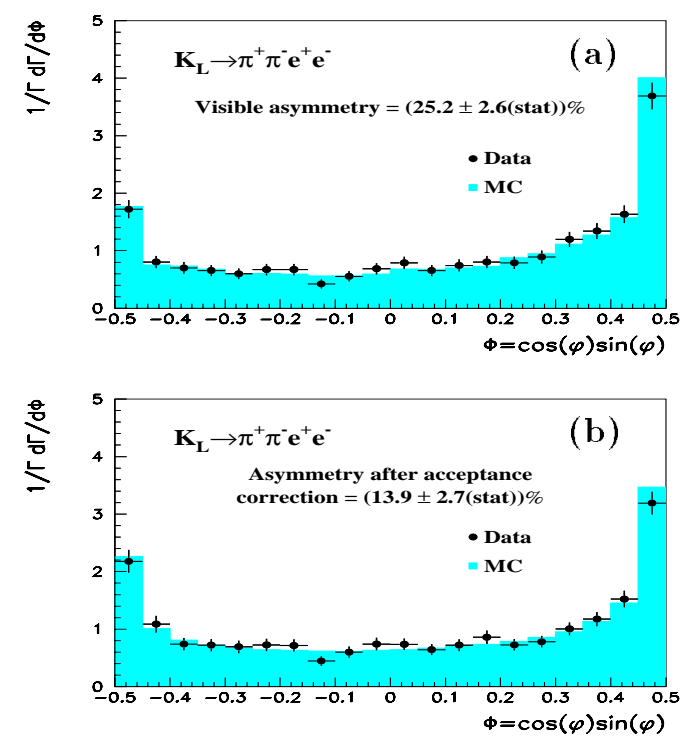

Figure 2. Angular distribution of $K_{L} \rightarrow$ $\pi^{+} \pi^{-} e^{+} e^{-}$events before acceptance correction (a) and after acceptance correction (b).

\section{2. $K_{S} \rightarrow \pi^{+} \pi^{-} e^{+} e^{-}$}

The $K_{S} \rightarrow \pi^{+} \pi^{-} e^{+} e^{-}$decay amplitude is largely dominated by the CP-even inner bremsstrahlung component and therefore no significant asymmetry in the $\phi$ distribution is expected in this case.

The first observation of this decay mode comes from a clean sample of 56 events obtained with the 1998 data. The analysis of this decay mode relies not only on the very good vertex resolution for $K_{S} / K_{L}$ separation but also on the $K_{S}$ tagging detector [8] which provides an extra factor of 20 in the background suppression from the $K_{L}$ beam.
Figure 3 shows the invariant mass $M_{\pi \pi e e}$ distribution for $K_{S} \rightarrow \pi^{+} \pi^{-} e^{+} e^{-}$candidates. The remaining contribution at low mass values is due to $K_{L} \rightarrow \pi^{+} \pi^{-} \pi_{D}^{0}$ decays where the $K_{L}$ originates from the $K_{S}$ target. Based on the 1998 data, the NA8 experiment has obtained a branching fraction value of $(4.5 \pm 0.7 \pm 0.4) \times 10^{-5}$ [9] The normalization events used for this measurement come from 105 fully reconstructed $K_{L} \rightarrow$ $\pi^{+} \pi^{-} \pi_{D}^{0} \rightarrow \pi^{+} \pi^{-} e^{+} e^{-} \gamma$ events originating from the $K_{S}$ target.

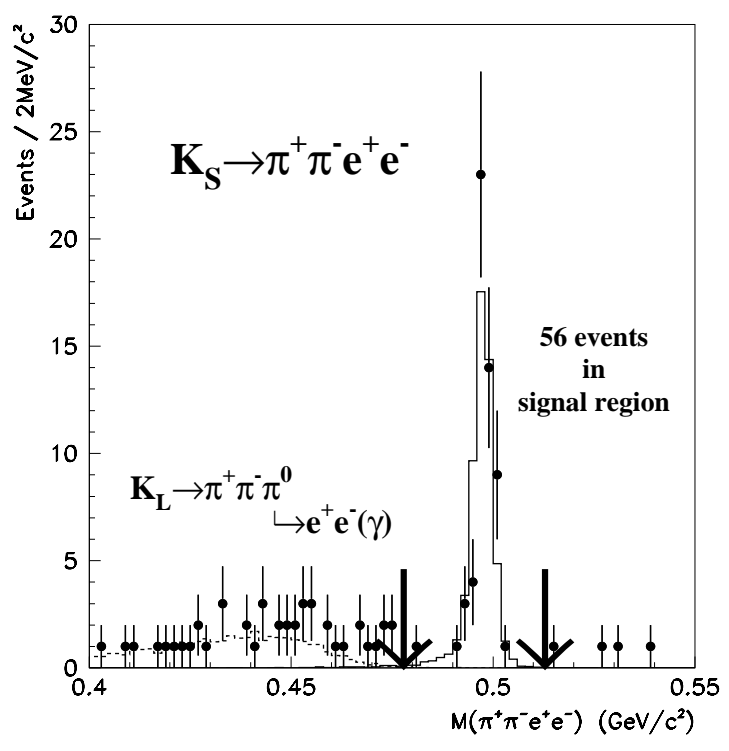

Figure 3. Invariant $\pi^{+} \pi^{-} e^{+} e^{-}$mass distribution for $K_{S} \rightarrow \pi^{+} \pi^{-} e^{+} e^{-}$candidates from 1998 data. The signal region is indicated by arrows.

\subsection{Radiative non-leptonic $K_{L}$ decays}

Several other rare $K_{L}$ decays have been investigated recently by NA48. For instance, the use of the four-track trigger has permitted the study of decays with four leptons in the final state: $K_{L} \rightarrow e^{+} e^{-} e^{+} e^{-}$and $K_{L} \rightarrow e^{+} e^{-} \mu^{+} \mu^{-}$.

These modes are particularly interesting for the understanding of the $K_{L} \gamma^{*} \gamma^{*}$ vertex. In 1999, 
the experiment has observed 132 and 19 events for the $K_{L} \rightarrow e^{+} e^{-} e^{+} e^{-}$and $K_{L} \rightarrow e^{+} e^{-} \mu^{+} \mu^{-}$ modes respectively. The preliminary value of the branching ratio for the decay into four electrons is $B R\left(K_{L} \rightarrow e^{+} e^{-} e^{+} e^{-}\right)=(3.67 \pm 0.32 \pm 0.24) \times$ $10^{-8}$. A branching ratio determination for the decay $K_{L} \rightarrow e^{+} e^{-} \mu^{+} \mu^{-}$is in progress and should be available soon.

The radiative $K_{L} \rightarrow e^{+} e^{-} \gamma \gamma$ decay mode is the most serious background to the very rare $K_{L} \rightarrow \pi^{0} e^{+} e^{-}$channel whose observation, predicted to occur at the $\sim \mathcal{O}\left(10^{-11}\right)$ level, would be of considerable interest for the study of direct $\mathrm{CP}$ violation.

From the data collected in 1997 and in 1998, the branching ratio of the $K_{L} \rightarrow e^{+} e^{-} \gamma \gamma$ decay mode has been measured to be $(6.32 \pm 0.31 \pm$ $0.35) \times 10^{-7}$ with a cut-off value of $5 \mathrm{MeV}$ on the photon energies in the kaon rest frame. This preliminary measurement, based on a sample of 492 candidates, is in good agreement with theoretical expectations [10].

The study of the $K_{L} \rightarrow \pi^{0} \gamma \gamma$ allows to test $\chi \mathrm{PT}$ predictions at $\mathcal{O}\left(p^{6}\right)$ and to better understand the role of vector mesons in weak transitions. A precise measurement of this decay channel provides also valuable information of the $\mathrm{CP}$ conserving part of the $K_{L} \rightarrow \pi^{0} e^{+} e^{-}$decay. Using a fraction of the 1998 data and the full data set of 1999 , the NA48 experiment has identified a sample of about 1400 events with a $2 \%$ background contamination. A preliminary branching ratio value for this decay mode is found to be $(1.51 \pm 0.05 \pm 0.20) \times 10^{-6}$, assuming an effective vector coupling $a_{v}$ of -0.45 .

The statistics and branching ratio values obtained recently for the above $K_{L}$ decays are summarized in Table 1.

\section{HIGH INTENSITY $K_{S}$ RUN}

During the 1999 run, a short period of 2 days was devoted to the investigation of rare $K_{S}$ and neutral hyperon decays with an intensity of the proton beam on the $K_{S}$ target that was increased by a factor of about 200 . The AKS converter which is used in the $\varepsilon^{\prime} / \varepsilon$ run to define precisely the beginning of the fiducial region for
$K_{S} \rightarrow \pi^{0} \pi^{0}$ decays was removed and the $K_{L}$ beam switched off. With these conditions, the instantaneous rates in the various NA48 detector elements were only slightly above the ones measured with the standard $\varepsilon^{\prime} / \varepsilon$ configuration. The total number of $K_{S}$ decays in the fiducial decay region of the experiment was about $4 \times 10^{8}$, equivalent to several years of operation with the standard $K_{L}+K_{S}$ beam setup. The amount of data collected during this short test allowed to improve substantially several measurements on rare $K_{S}$ and neutral hyperon decays.
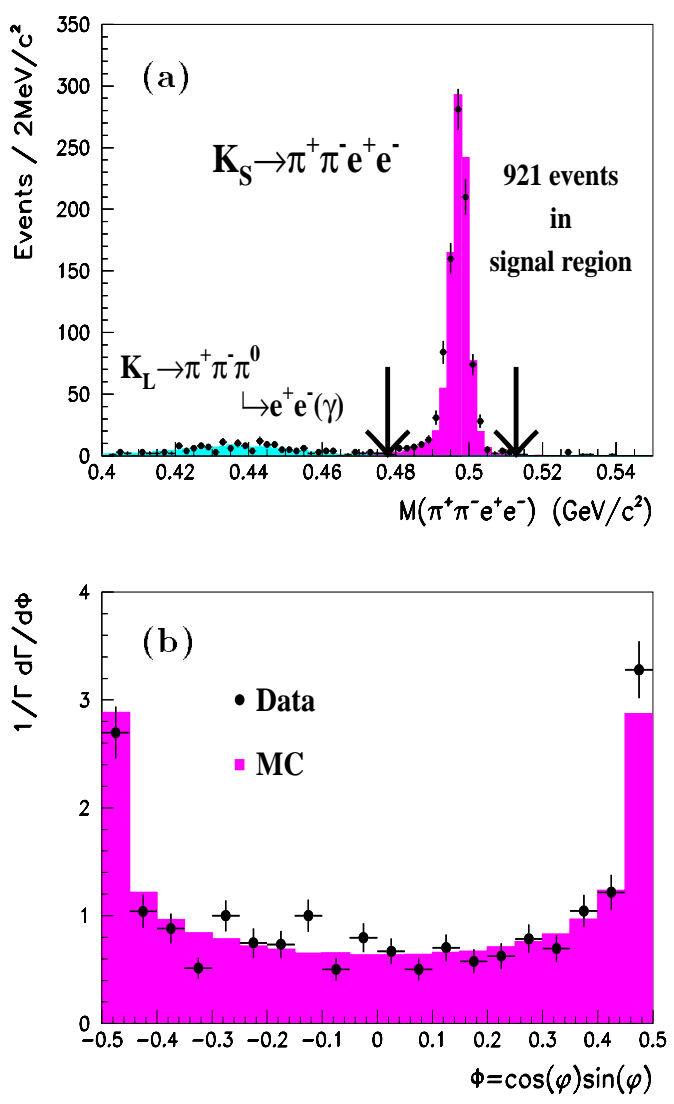

Figure 4. Invariant $\pi^{+} \pi^{-} e^{+} e^{-}$mass (a) and angular (b) distributions for $K_{S} \rightarrow \pi^{+} \pi^{-} e^{+} e^{-}$candidates. 
Table 1

Rare $K_{L}$ decay modes.

\begin{tabular}{lcrcc}
\hline \multicolumn{1}{c}{ Channel } & Year & Events & S/B & BR \\
\hline$K_{L} \rightarrow \pi^{+} \pi^{-} e^{+} e^{-}$ & $1998+1999$ & 1337 & 38 & $(3.1 \pm 0.1 \pm 0.2) \times 10^{-7}$ \\
$K_{L} \rightarrow e^{+} e^{-} e^{+} e^{-}$ & 1999 & 132 & 40 & $(3.67 \pm 0.32 \pm 0.24) \times 10^{-8}$ \\
$K_{L} \rightarrow e^{+} e^{-} \mu^{+} \mu^{-}$ & 1999 & 19 & - & - \\
$K_{L} \rightarrow e^{+} e^{-} \gamma \gamma$ & $1997+1998$ & 492 & 20 & $(6.32 \pm 0.31 \pm 0.35) \times 10^{-7}$ \\
$K_{L} \rightarrow \pi^{0} \gamma \gamma$ & 1998 (part of) +1999 & 1397 & 47 & $(1.51 \pm 0.05 \pm 0.20) \times 10^{-6}$ \\
\hline
\end{tabular}

3.1. Asymmetry in $K_{S} \rightarrow \pi^{+} \pi^{-} e^{+} e^{-}$decays The statistical gain of $\sim 4$ in the number of $K_{S} \rightarrow \pi^{+} \pi^{-} e^{+} e^{-}$events with respect to the total sample collected in 1998 and 1999 with the standard $\varepsilon^{\prime} / \varepsilon$ configuration allowed to perform a measurement of the $\phi$ asymmetry at a few percent level. Figure 4 shows the invariant mass $M_{\pi \pi e e}$ and $\phi$ distributions for $K_{S} \rightarrow \pi^{+} \pi^{-} e^{+} e^{-}$ candidates using the full 1998+1999 data sample. With a grand total of 921 signal events, the asymmmetry is measured to be $\mathcal{A}_{S}=(-0.2 \pm$ $3.4 \pm 1.4) \%$, compatible with zero. The value obtained for the branching ratio is $B R\left(K_{S} \rightarrow\right.$ $\left.\pi^{+} \pi^{-} e^{+} e^{-}\right)=(4.3 \pm 0.2 \pm 0.3) \times 10^{-5}$. This result translates into a contribution of $B R\left(K_{L}^{I B} \rightarrow\right.$ $\left.\pi^{+} \pi^{-} e^{+} e^{-}\right)=(1.3 \pm 0.1) \times 10^{-7}$ for the inner bremsstrahlung part of the $K_{L} \rightarrow \pi^{+} \pi^{-} e^{+} e^{-}$ process, in very good agreement with theoretical expectations $[3,4]$.

\section{2. $K_{S} \rightarrow \gamma \gamma$}

The $K_{S} \rightarrow \gamma \gamma$ decay channel, which receives negligible short-distance contributions, is particularly interesting from the theoretical point of view. Within the framework of chiral lagrangians, the one-loop contribution to $K_{S} \rightarrow \gamma \gamma$ is finite and gives an unambiguous prediction of the branching ratio. A precise measurement of $B R\left(K_{S} \rightarrow \gamma \gamma\right)$ would therefore provide a valuable test of $\chi P T$ and shed light on contributions from high-order loop corrections.

The current experimental determination of the branching ratio comes from the measurement published in 1995 by the NA31 Collaboration [11]: $B R\left(K_{S} \rightarrow \gamma \gamma\right)=(2.4 \pm 0.9) \times 10^{-6}$ to be compared to the theoretical prediction of $2.25 \times 10^{-6}$ [12].

Owing to the large background contribution from $K_{S} \rightarrow \pi^{0} \pi^{0}$ decays, the study of the $K_{S} \rightarrow$ $\gamma \gamma$ decay by NA48 can only be carried out in a very restricted decay region, close to the exit face of the beam collimators. The maximum value of the invariant mass for a $\gamma \gamma$ pair originating from $K_{S} \rightarrow \pi^{0} \pi^{0}$ decays is about $458 \mathrm{MeV} / \mathrm{c}^{2}$. This produces an apparent neutral vertex shift of about $9 \mathrm{~m}$, leaving the beginning of the decay region populated mainly by $K_{L, S} \rightarrow \gamma \gamma$.

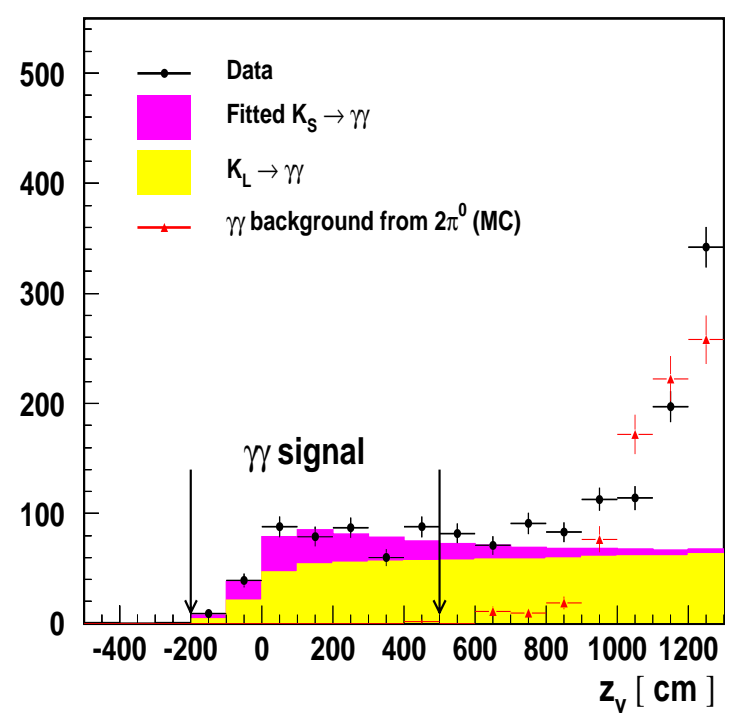

Figure 5. $Z_{\text {vertex }}$ distribution for $\gamma \gamma$ events. The contributions from $K_{S} \rightarrow \gamma \gamma$ and $K_{L} \rightarrow \gamma \gamma$ are shown together with the Monte Carlo prediction for the $\gamma \gamma$ background from $2 \pi^{0}$. 
From the two-day long test run performed in 1999, $450 K^{0} \rightarrow \gamma \gamma$ candidates with energy between 60 and $170 \mathrm{GeV}$ have been identified in the $-2 \mathrm{~m}<Z_{\text {vertex }}<5 \mathrm{~m}$ region. Figure 5 shows the distribution of the reconstructed longitudinal vertex position after all other analysis cuts have been applied. The background contamination is estimated to be $13 \pm 8$ events, mainly due to $\Lambda \rightarrow n \pi^{0}$ and $K_{S} \rightarrow \pi^{0} \pi^{0}$.

Using the existing branching ratio value for $K_{L} \rightarrow \gamma \gamma$ and a binned Maximum Likelihood method, $149 \pm 21 K_{S} \rightarrow \gamma \gamma$ events have been estimated to lie in the signal region.

The NA48 experiment obtains $B R\left(K_{S} \rightarrow \gamma \gamma\right)$ $=(2.58 \pm 0.36 \pm 0.22) \times 10^{-6}[13]$ using the abundant $K_{S} \rightarrow \pi^{0} \pi^{0}$ decays as normalization sample. This result yields

$\frac{\Gamma\left(K_{S} \rightarrow \gamma \gamma\right)}{\Gamma\left(K_{L} \rightarrow \gamma \gamma\right)}=(2.53 \pm 0.35 \pm 0.22)$.

\subsection{Search for $K_{S} \rightarrow \pi^{0} e^{+} e^{-}$}

The CP conserving $K_{S} \rightarrow \pi^{0} e^{+} e^{-}$decay is well known to be dominated by long-distance dynamics through one-photon exchange. However, theoretical predictions of the branching ratio suffer from strong model-dependent assumptions. G. D'Ambrosio et al. [14] have calculated the branching ratio beyond leading order in the chiral expansion and found

$B R\left(K_{S} \rightarrow \pi^{0} e^{+} e^{-}\right) \sim 5.2 a_{S}^{2} \times 10^{-9}$

where $a_{S}$ is a parameter of $\mathcal{O}(1)$ to be determined experimentally. The best upper limit on $B R\left(K_{S} \rightarrow \pi^{0} e^{+} e^{-}\right)$comes from the NA31 Collaboration [15] which obtained $B R\left(K_{S} \rightarrow\right.$ $\left.\pi^{0} e^{+} e^{-}\right)<1.1 \times 10^{-6}$ at $90 \% \mathrm{CL}$.

A more precise measurement of the branching ratio is of considerable interest in order to improve the limit on the indirect $\mathrm{CP}$ violating contribution in $K_{L} \rightarrow \pi^{0} e^{+} e^{-}$. While the CP conserving part of this decay mode is dominated by the two-photon process $K_{L} \rightarrow \pi^{0} \gamma^{*} \gamma^{*}$ and predicted to occur at the level of a few $10^{-12}$, the $\mathrm{CP}$ violating term (CPV) receives contributions from both direct and indirect $\mathrm{CP}$ violation:

$10^{12} \times B R\left(K_{L} \rightarrow \pi^{0} e^{+} e^{-}\right)_{C P V}=$ $\left[15.3 a_{S}^{2}-6.8 \frac{\operatorname{Im}\left(\lambda_{t}\right)}{10^{-4}} a_{S}+2.8\left(\frac{\operatorname{Im}\left(\lambda_{t}\right)}{10^{-4}}\right)^{2}\right]$

where $\operatorname{Im}\left(\lambda_{t}\right)=\operatorname{Im}\left(V_{t d} V_{t s}^{*}\right) \sim \mathcal{O}\left(10^{-4}\right)$ describes the short distance $C P$ violation and $a_{S}$ gives the strength of indirect $\mathrm{CP}$ violation.

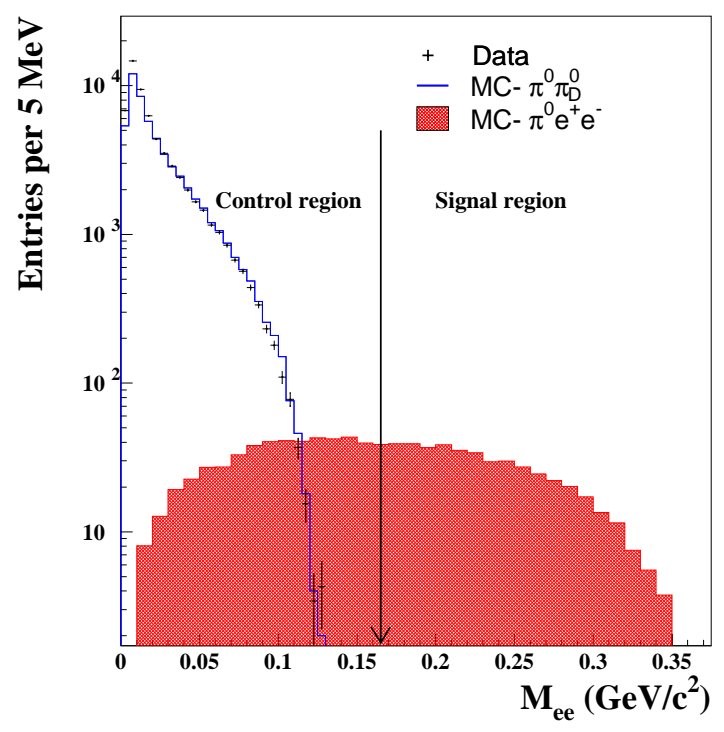

Figure 6. Invariant mass distribution of the $e^{+} e^{-}$ pair for data (dots) and for $\pi^{0} \pi_{D}^{0}$ Monte Carlo events (histogram). The shaded area is the expected distribution (arbitrary normalization) for $K_{S} \rightarrow \pi^{0} e^{+} e^{-}$decays.

With values of $a_{S}<-0.5$ or $a_{S}>1.0$, the rate of $K_{L} \rightarrow \pi^{0} e^{+} e^{-}$would be mainly due to $C P$ violating effects and the determination of $B R\left(K_{S} \rightarrow \pi^{0} e^{+} e^{-}\right)$could become accessible to experiment.

During the short test performed with a high intensity $K_{S}$ beam, the NA48 experiment has improved the limit on $B R\left(K_{S} \rightarrow \pi^{0} e^{+} e^{-}\right)$by almost a factor of 10 .

The most severe source of background clearly comes from $K_{S} \rightarrow \pi^{0} \pi^{0}$ decays with the subse- 
quent Dalitz decay of one of the two neutral pions. These unwanted events can be strongly suppressed by requiring a cut on the invariant mass of the electron pair above the $m_{\pi^{0}}$ kinematical limit in order to reject possible background contributions due to resolution effects or interactions in the detector material.

Figure 6 shows the $M_{e e}$ distribution for $\pi^{0} e^{+} e^{-}$ candidates in the final sample together with the Monte Carlo distribution for background and signal events. The observed $M_{e e}$ distribution is very well accounted for by background events due to $K_{S} \rightarrow \pi^{0} \pi_{D}^{0}$ decays. Above the $M_{e e}=165 \mathrm{MeV} / \mathrm{c}^{2}$ value which defines the signal region, no candidate is observed. Contaminations from $K_{S} \rightarrow \pi^{0} \pi_{D}^{0}$ or $K_{S} \rightarrow \pi_{D}^{0} \pi_{D}^{0}$ decays are estimated to be less than 0.03 event for each channel. Using fully reconstructed $K_{S} \rightarrow$ $\pi^{0} \pi_{D}^{0} \rightarrow \pi^{0} e^{+} e^{-} \gamma$ events, a preliminary upper limit of $1.6 \times 10^{-7}$ at $90 \% \mathrm{CL}$ on the branching ratio for $K_{S} \rightarrow \pi^{0} e^{+} e^{-}$is obtained. This result correspond to an upper limit of $4.6 \times 10^{-10}$ at $90 \%$ CL on the branching ratio for the indirect CP violating part of the $K_{L} \rightarrow \pi^{0} e^{+} e^{-}$decay.

\subsection{Neutral hyperon decays}

Neutral hyperon decays have been investigated using a specialized charged trigger logic that enriched the sample of $\Lambda$ decays. It combines cuts on the invariant $\pi^{+} \pi^{-}$mass and $p^{+} / p^{-}$momenta ratio to reject a large fraction of the $K_{S} \rightarrow$ $\pi^{+} \pi^{-}$events (Figure 7 ). During the short test run, about $41 \times 10^{6} 2$-track events were recorded among which $17 \times 10^{6}$ were identified as $\Lambda \rightarrow p \pi^{-}$ decays and $2 \times 10^{6}$ as $\bar{\Lambda} \rightarrow \bar{p} \pi^{+}$. Most of the studies performed with these data sets are related to the radiative $\Xi^{0} \rightarrow \Lambda \gamma$ and $\Xi^{0} \rightarrow \Sigma^{0} \gamma$ decays as well as the semileptonic decays of the $\Xi^{0}$ particle. For the branching ratio measurement of the radiative $\Xi^{0}$ decays, the abundant $\Xi^{0} \rightarrow \Lambda \pi^{0}$ channel was used.

The NA48 experiment has observed $497 \Xi^{0} \rightarrow$ $\Lambda \gamma$ with negligible background yielding a preliminary branching ratio determination of $(1.9 \pm 0.1 \pm$ $0.2) \times 10^{-3}$. The systematic uncertainty is dominated by the $\Xi^{0}$ polarization and the decay asymmetry. Figure 8 shows the invariant $\Lambda \gamma$ mass distribution for the observed events.

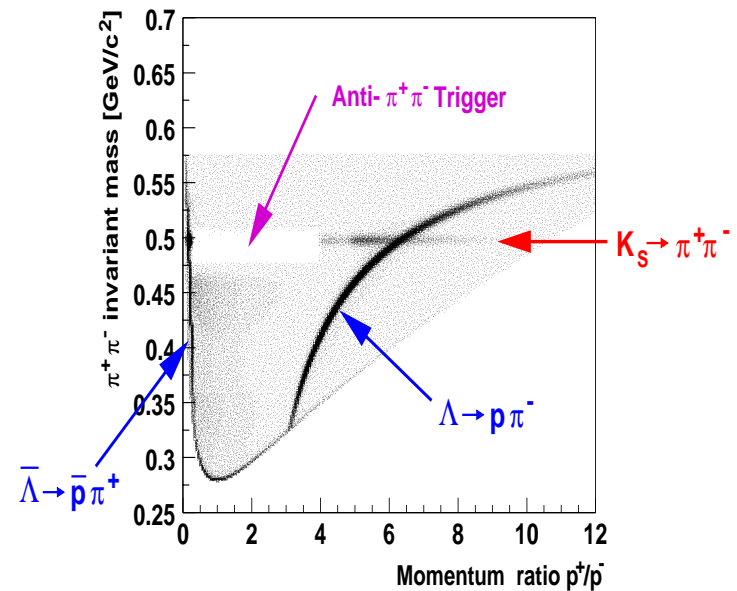

Figure 7. Invariant $\pi^{+} \pi^{-}$mass as a function of the momentum ratio of tracks. The veto region against $K_{S} \rightarrow \pi^{+} \pi^{-}$decays appears in the figure as an empty rectangular box.

The signature of the $\Xi^{0} \rightarrow \Sigma^{0} \gamma$ channel is obtained by looking at the $\Xi^{0} \rightarrow \Sigma^{0} \gamma \rightarrow \Lambda \gamma \gamma$ decay chain. The detailed analysis of this decay is discussed by A. Zinchenko [16].

The semileptonic $\Xi^{0} \rightarrow \Sigma^{+} e^{-} \bar{\nu}_{e}$ decay is the direct analogue to the neutron $\beta$ decay. The investigation of this mode allows to study SU(3) symmetry breaking effects. The main background sources are the $\Lambda \rightarrow p \pi^{-}$and $\Lambda \rightarrow p e^{-} \bar{\nu}_{e}$ decays. NA48 has observed about 60 events which should allow soon a measurement of its branching ratio with a precision of about $15 \%$.

Finally, a search for the suppressed $\Delta S=2$ $\Xi^{0} \rightarrow p \pi^{-}$decay channel has been performed. No event of this type has been found in our data set. NA48 should be able to improve the present limit of $3.6 \times 10^{-5}$ at $90 \% \mathrm{CL}$ [17] by about a factor of 10 .

\section{HIGH INTENSITY $K_{S}$ RUN IN 2000}

Due to the implosion in November 1999 of the carbon fibre beam pipe which traverses the NA48 detector, all four drift chambers were seriously damaged. The repair of the detectors is now in 


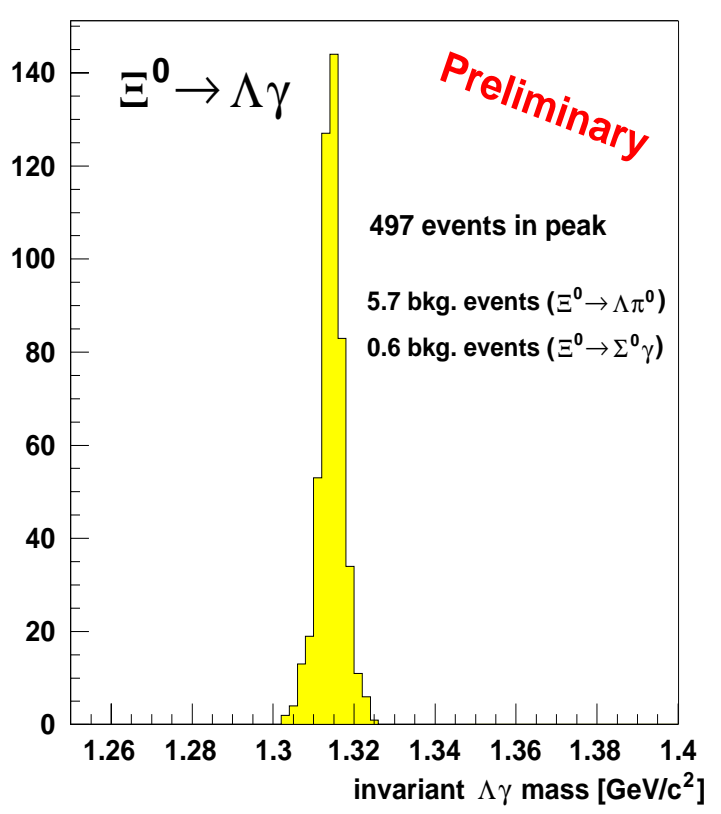

Figure 8. Invariant $\Lambda \gamma$ mass distribution for $\Xi^{0} \rightarrow \Lambda \gamma$ decays.

progress and the spectrometer is scheduled to be operational by summer 2001. As a consequence, the NA48 experiment took data in 2000 without magnetic spectrometer, recording only neutral decays. A continuous vacuum could however be establisehd from the $K_{S}$ target to the end of the He tank which contains, in normal conditions, the drift chambers. About 40 days were spent in 2000 with a high intensity $K_{S}$ beam in order to investigate rare decays into purely neutral modes. The proton intensity on the $K_{S}$ target was about $9 \times 10^{9}$ per SPS pulse of $3.2 \mathrm{~s}$ every $14.4 \mathrm{~s}$. The momentum of the proton beam was reduced from 450 $\mathrm{GeV} / \mathrm{c}$ to $400 \mathrm{GeV} / \mathrm{c}$ and the kaon production angle was decreased from $4.2 \mathrm{mrad}$ to about $3 \mathrm{mrad}$ in order to compensate the beam momentum loss. The total number of $K_{S}$ decaying in the fiducial volume of the experiment was about $1 \times 10^{10}$. Table 2 shows, for different $K_{S}$ decay channels, the expected total number of reconstructed events in this run.

One very challenging decay to investigate experimentally is the $K_{S} \rightarrow \pi^{0} \pi^{0} \pi^{0}$ mode which has a very small branching ratio. As the $3 \pi^{0}$ state is pure $\mathrm{CP}=-1$, the observation of the $K_{S} \rightarrow$ $\pi^{0} \pi^{0} \pi^{0}$ decay is a clear signature of CP violation. The strength of this violation is parametrized by

$\eta_{000}=\frac{A\left(K_{S} \rightarrow \pi^{0} \pi^{0} \pi^{0}\right)}{A\left(K_{L} \rightarrow \pi^{0} \pi^{0} \pi^{0}\right)}$.

Currently, the best measurement of $\eta_{000}$ is the one reported by the CPLEAR Collaboration [18]: $\operatorname{Re}\left(\eta_{000}\right)=(0.18 \pm 0.15)$ and $\operatorname{Im}\left(\eta_{000}\right)=(0.15 \pm$ $0.20)$, corresponding to a limit on the branching ratio of $B R\left(K_{S} \rightarrow \pi^{0} \pi^{0} \pi^{0}\right)<1.9 \times 10^{-5}$ at $90 \%$ CL. More recently, the SND experiment [19] has lowered the limit on the branching fraction to $1.4 \times 10^{-5}$ at $90 \% \mathrm{CL}$.

A significantly more precise measurement of $\eta_{000}$ would be of considerable interest to constrain tests of CPT in the neutral kaon sector. If unitarity is assumed, it is then possible to measure the CPT violation parameter $\operatorname{Im}(\delta)$ through the BellSteinberger relation which relates $K_{S}$ and $K_{L}$ decay amplitudes into all final states:

$\left(1+i \tan \phi_{S W}\right)[\operatorname{Re}(\varepsilon)-i \operatorname{Im}(\delta)]=$

$$
\sum A_{f L} A_{f S}^{*} / \Gamma_{S}
$$

where $\varepsilon$ describes CP violation with CPT conservation.

The best determinations of $\operatorname{Im}(\delta)$ and $\operatorname{Re}(\varepsilon)$ come from the CPLEAR experiment [20] which has obtained, using the unitarity assumption only: $\operatorname{Im}(\delta)=(2.4 \pm 5.0) \times 10^{-5}$ and $\operatorname{Re}(\varepsilon)=$ $(164.9 \pm 2.5) \times 10^{-5}$. As the uncertainty on both parameters is dominated by the one on $\eta_{000}$, a better limit on $\eta_{000}$ would improve the CPT test.

The NA48 method to extract $\eta_{000}$ is based on the measurement of the $K_{S}-K_{L}$ interference term in the neutral kaon decay probability into $3 \pi^{0}$ as a function of proper decay time:

$$
\begin{aligned}
& I_{000}(t) \propto e^{-t / \tau_{L}}+\left|\eta_{000}\right|^{2} e^{-t / \tau_{S}}+ \\
& 2 D\left|\eta_{000}\right| e^{\frac{-t}{2}\left(\frac{1}{\tau_{S}}+\frac{1}{\tau_{L}}\right)} \cos \left(\Delta m t+\phi_{000}\right)
\end{aligned}
$$

where $D=\frac{N_{K}^{0}-N_{\bar{K}^{0}}^{0}}{N_{K}^{0}+N_{\bar{K}^{0}}^{0}}$ is $\sim 0.3$ for NA48.

The observation of such an interference term is difficult since the effect is superimposed on the 
Table 2

Rare $K_{S}$ decay modes.

\begin{tabular}{lccr}
\hline Channel & $\begin{array}{c}\text { BR } \\
\text { (Experiment[21]) }\end{array}$ & $\begin{array}{c}\text { BR } \\
\text { (Theory[22]) }\end{array}$ & $\begin{array}{c}\text { Events } \\
/ 10^{10} K_{S}\end{array}$ \\
\hline$K_{S} \rightarrow \gamma \gamma$ & $(2.4 \pm 0.9) \times 10^{-6}$ & $2.1 \times 10^{-6}$ & 8000 \\
$K_{S} \rightarrow \pi^{0} \gamma \gamma$ & - & $3.8 \times 10^{-8}$ & 38 \\
$K_{S} \rightarrow \pi^{0} \pi^{0} \gamma \gamma$ & - & $5.6 \times 10^{-9}$ & 2 \\
$K_{S} \rightarrow \pi^{0} \pi^{0} \pi^{0}$ & $<1.4 \times 10^{-5}(90 \% \mathrm{CL})$ & $2.5 \times 10^{-9}$ & 1 \\
$K_{S} \rightarrow \pi^{0} \pi^{0} \gamma$ & - & $1.7 \times 10^{-11}$ & 0 \\
\hline
\end{tabular}

very large and flat $K_{L} \rightarrow \pi^{0} \pi^{0} \pi^{0}$ component and is mostly contained in the $\sim 1.2 c \tau_{S}$ region between the $K_{S}$ production target and the collimator end. This loss of sensitivity is however partly compensated by the very good resolution on the proper decay time $\left(\sim 0.1 c \tau_{S}\right)$ provided by the use of high energy beams and high resolution calorimetry.

In order to significantly improve the present limits on $\eta_{000}$, high statistics samples of $K^{0} \rightarrow$ $\pi^{0} \pi^{0} \pi^{0}$ decays must be collected within a few $K_{S}$ lifetimes from the target $\left(<5 c \tau_{S}\right)$. Moreover, an excellent knowledge of the detector acceptance is required to reach a high sensitivity to $\eta_{000}$.

For the 2000 data taking period, the estimated number of fully reconstructed events in the 90-140 GeV kaon energy range is about $1 \times 10^{6} / c \tau_{S}$. A much larger sample of $K_{L} \rightarrow \pi^{0} \pi^{0} \pi^{0}$ decays with the $K_{L}$ beam was also collected during part of the 2000 run that was devoted to the $\varepsilon^{\prime} / \varepsilon$ systematic studies. These events can be used to cross-check the Monte Carlo simulation for the acceptance correction. A very preliminary analysis of the 2000 data indicates that a limit of about $3 \%$ on $\operatorname{Re}\left(\eta_{000}\right)$ and $\operatorname{Im}\left(\eta_{000}\right)$ could be reached, thus improving significantly the current measurement of $\eta_{000}$ and the CPT test.

\section{PROSPECTS FOR 2002}

The NA48 Collaboration has recently proposed to use a modified $K_{S}$ beam line in order to investigate $K_{S}$ and neutral hyperon decays with high sensitivities [23]. This physics programme could start in 2002 after the completion of the $\operatorname{Re}\left(\varepsilon^{\prime} / \varepsilon\right)$ measurement in 2001.
Minor modifications to the present $K_{S}$ beam line are foreseen in order to optimize, at low cost, the experimental conditions. The proposed changes include, for instance, the possibility to exploit a longer SPS duty cycle, with a spill duration of $5.2 \mathrm{~s}$ every $16.8 \mathrm{~s}$. The beam momentum and the kaon production angle would be tuned to respectively $400 \mathrm{GeV} / \mathrm{c}$ and $2.5 \mathrm{mrad}$. It is also proposed to use a sweeping magnet at the end of the $K_{S}$ collimator $(\oint B \cdot d l \simeq 0.6 \mathrm{Tm})$ in order to reject photon conversions and to operate the $K_{S}$ sweeping magnet at the maximum $7.5 \mathrm{Tm}$ strength.

These measures should allow the primary proton flux to be increased to $1 \times 10^{10}$ per pulse yielding about $3 \times 10^{10} K_{S}$ decays per year in the fiducial volume of the experiment. Instantaneous rates in the NA48 detector are expected to be $\sim 1.5$ the ones measured in the $\varepsilon^{\prime} / \varepsilon$ operation mode. For $K_{S} \rightarrow \pi^{0} e^{+} e^{-}$decays, the SES would correspond to $6 \times 10^{-10}$ assuming an overall acceptance of $5 \%$.

In addition to the replacement of the damaged beam pipe and the four drift chambers, some modest changes in the calorimeter and spectrometer read-out systems are also envisaged in order to increase the present trigger rate capability of the experiment from 7.5 to $15 \mathrm{kHz}$. This is particularly important for the study of the copious neutral hyperon decay samples that will be available.

One of the main goals of this physics programme is the search for the $K_{S} \rightarrow \pi^{0} e^{+} e^{-}$ decay. The expected signal in this case is 7 events/year for a branching fraction of $5 \times 10^{-9}$. The main source of background is due to $K_{S} \rightarrow$ 
$\pi^{0} \pi_{D}^{0}$. This contamination is estimated to be less than 0.3 event/year according to a Monte Carlo simulation of events generated with an invariant mass $M_{e e}$ greater than $90 \mathrm{MeV} / \mathrm{c}^{2}$. Other backgrounds like $K_{S} \rightarrow \pi_{D}^{0} \pi_{D}^{0}$ or $K_{L, S} \rightarrow e^{+} e^{-} \gamma \gamma$ should be negligible.

The $K_{S} \rightarrow \pi^{0} \mu^{+} \mu^{-}$decay will also be investigated but its branching ratio is about five times smaller than the one of $K_{S} \rightarrow \pi^{0} e^{+} e^{-}$and the background sources are of different nature (e.g. $K_{L} \rightarrow \pi^{+} \pi^{-} \pi^{0}$ with two decaying pions).

As far as the $K_{S} \rightarrow \pi^{0} \pi^{0} \pi^{0}$ channel is concerned, the experiment sensitivity should allow to put a bound on $\eta_{000}$ of about $1 \%$ and provide a further test of CPT.

Several less rare $K_{S}$ decays will be studied with high statistical precision. For example, about $24 \mathrm{k}$ $K_{S} \rightarrow \gamma \gamma$ decays are expected to be collected in one year with a similar $K_{L} \rightarrow \gamma \gamma$ background level per $K_{S}$ lifetime. The copious $K_{S} \rightarrow \pi^{+} \pi^{-} \gamma$ and $K_{S} \rightarrow \pi^{+} \pi^{-} e^{+} e^{-}$decay channels will be measured with about $5 \times 10^{6}$ and $5 \times 10^{4}$ events per year respectively. The study of these modes will provide information on form factors in the direct emission process.

The sensitivity of the experiment should also allow the observation of the rare $K_{S} \rightarrow \pi^{0} \gamma \gamma$, $K_{S} \rightarrow \pi^{0} \pi^{0} \gamma \gamma, K_{S} \rightarrow e^{+} e^{-} \gamma$ or $K_{S} \rightarrow \mu^{+} \mu^{-} \gamma$ decays.

The $\beta$ decay of the $\Xi^{0}$ hyperon, which has a branching ratio of $2.5 \times 10^{-4}$, can be detected in NA48 through the decay $\Xi^{0} \rightarrow \Sigma^{+} e^{-} \bar{\nu}$ followed by $\Sigma^{+} \rightarrow p \pi^{0}$. About $25 \mathrm{k}$ events are expected to be collected in the proposed run. These events will be used for a consitency check of SU(3) symmetry and the Cabbibo model in hyperon decays.

The radiative decay modes $\Xi^{0} \rightarrow \Lambda \gamma$ and $\Xi^{0} \rightarrow \Sigma^{0} \gamma$ will be investigated with an expected statistical gain of about 100 with respect to previous NA48 measurements. The uncertainty on the branching ratio determinations for these two modes will be about $5 \%$. Measurements of decay asymmetries with improved precision will also be obtained.

The NA48 detector with its high resolution photon detector will be able to reduce the experimental uncertainty on the $\Xi^{0}$ mass to $0.1 \mathrm{MeV}$, at the level of the current error on the $\Xi^{-}$mass.
Finally, NA48 will search for the $\Delta S=2$ $\Xi^{0} \rightarrow p \pi^{-}$process and improve the present experimental limit on the branching ratio by about two orders of magnitude.

\section{NA48 AND BEYOND}

The recently published values of $\operatorname{Re}\left(\varepsilon^{\prime} / \varepsilon\right)$ by $\mathrm{KTeV}$ [24] and NA48 [25] have confirmed the existence of direct $\mathrm{CP}$ violation in $K^{0} \rightarrow 2 \pi$ decays. A measurement of direct $\mathrm{CP}$ violation in other processes is of great importance in order to better understand the origin of this effect.

The NA48 Collaboration has proposed to look for a manifestation of $\mathrm{CP}$ violation through the measurement of the Dalitz plot decay parameters in $K^{ \pm} \rightarrow \pi^{+} \pi^{-} \pi^{ \pm}$using an extended NA48 setup [26].

The matrix element for the decays $K^{ \pm} \rightarrow$ $\pi^{+} \pi^{-} \pi^{ \pm}$can be parametrized by

$|M(u, v)|^{2} \propto 1+g u+h u^{2}+k v^{2}$

where $u=\left(s_{3}-s_{0}\right) / m_{\pi}^{2}, v=\left(s_{1}-s_{2}\right) / m_{\pi}^{2}, s_{0}=$ $\left(s_{1}+s_{2}+s_{3}\right) / 3$ and $s_{i}=\left(P_{K}-P_{i}\right)^{2}, P_{K}$ and $P_{i}$ are the four-momenta of the kaon and of the pion ( $i=3$ for the odd pion).

If CP conservation holds, then coefficients $g, h$ and $k$ are the same for $K^{+}$as for $K^{-}$decays. A measurement of direct $\mathrm{CP}$ violation can be obtained through the observation of a non-zero value for the asymmetry

$A_{g}=\frac{\left(g^{+}-g^{-}\right)}{\left(g^{+}+g^{-}\right)}$.

Theoretical predictions for $A_{g}$ in the framework of the Standard Model are in the $\mathcal{O}\left(10^{-6}\right)$ $\mathcal{O}\left(10^{-4}\right)$ range $[27,28]$. However, some supersymmetric models could give a value as high as $10^{-4}$ [29]. The best measurement of $A_{g}$ comes from an old experiment performed in 1970 [30] which obtained $A_{g}=(-7.0 \pm 5.3) \times 10^{-3}$.

The proposed experiment would use simultaneous $K^{+}$and $K^{-}$beams with a central momentum value of $60 \mathrm{GeV} / \mathrm{c}$ and a momentum bite of $\pm(10-20) \%$. Both charged beams would be selected with the same geometrical acceptance and be directed along a common line pointing towards the NA48 detector. To accomodate these features, a modification of the present NA48 beam 
elements downstream of the $K_{L}$ target would therefore be necessary. These changes could be ready in 2003 after the completion of the physics programme with a high intensity $K_{S}$ beam.

With an average proton beam intensity of about $1 \times 10^{12} /$ spill on the production target, NA48 could collect more than $2 \times 10^{9} K^{ \pm} \rightarrow$ $\pi^{+} \pi^{-} \pi^{ \pm}$decays per year and measure $A_{g}$ with a precision better than $2 \times 10^{-4}$. CP violation can also be investigated in decays like $K^{ \pm} \rightarrow \pi^{ \pm} \pi^{0} \pi^{0}$ or $K^{ \pm} \rightarrow \pi^{ \pm} \pi^{0} \gamma$. The expected precision on the asymmetry is better than $4 \times 10^{-4}$ for the $K^{ \pm} \rightarrow \pi^{ \pm} \pi^{0} \pi^{0}$ while an upper limit at the level of $10^{-2}$ could be obtained for $K^{ \pm} \rightarrow \pi^{ \pm} \pi^{0} \gamma$ decays.

The use of intense charged kaon beams would also allow to study in a clean way the $\pi^{+} \pi^{-}$interaction at low energy via the measurement of the $K^{ \pm} \rightarrow \pi^{+} \pi^{-} e^{ \pm} \nu(\bar{\nu})$ decays $\left(B R=3.9 \times 10^{-5}\right)$. As no other hadron is present in the final state, the extraction of the $\pi^{+} \pi^{-}$elastic scattering lengths $a_{0}^{0}$ and $a_{0}^{2}$ can be performed in a clean way. A precise measurement of $a_{0}^{0}$ to better than 0.01 would allow to obtain valuable information on the size of the single flavor condensate in the QCD vacuum and shed more light on the mechanism of spontaneous chiral symmetry breaking.

The current experimental value of $a_{0}^{0}$ comes from the 1977 Geneva-Saclay experiment [31]: $a_{0}^{0}=0.263 \pm 0.052$ based on a sample of $30 \mathrm{k}$ events. More recently, the BNL E865 experiment [32] has obtained the preliminary value $a_{0}^{0}=0.235 \pm 0.013($ stat $)$ with a sample of about $400 \mathrm{k} K_{e 4}$ decays.

The NA48 experiment aims at a precise measurement of $a_{0}^{0}$ with a statistical precision of about 0.007 . This result can be obtained in one year with $1 \times 10^{6}$ detected $K^{ \pm} \rightarrow \pi^{+} \pi^{-} e^{ \pm} \nu(\bar{\nu})$ decays. To achieve this goal, the NA48 would rely not only on the very good resolutions of the magnetic spectrometer and the LKr calorimeter but also on the use of the TRD to reduce pion contamination from $K^{ \pm} \rightarrow \pi^{+} \pi^{-} \pi^{ \pm}$decays at the level of a few $10^{-6}$. Furthermore, it is envisaged to use on the $K^{+}$and $K^{-}$beam lines a beam spectrometer made of MICROMEGAS-type detectors [33] in order to measure the momentum of the incident charged kaons with a precision of about $1 \%$

Several other interesting rare decays (e.g. $K^{ \pm} \rightarrow \pi^{ \pm} e^{+} e^{-}$) could also be investigated by the proposed experiment with significantly improved sensitivities.

\section{CONCLUSION}

The investigation of rare decays by NA48, concurrently with the measurement of $\operatorname{Re}\left(\varepsilon^{\prime} / \varepsilon\right)$, is providing interesting physics results for the understanding of chiral dynamics and CP Violation in the neutral kaon sector.

The high intensity $K_{S}$ beam test performed in 1999 together with the successful 2000 run have demonstrated clearly the capability of the NA48 experiment to investigate, in the near future, rare $K_{S}$ and neutral hyperon decays with high sensitivities.

Two proposals have been submitted recently to the CERN SPS Committee for the continuation of the NA48 experiment with high intensity $K_{S}$ and charged kaon beams. The proposed experimental programmes aim at exploring in a very competitive way a rich domain of kaon physics.

\section{REFERENCES}

1. C. Biino et al., Proceedings of the 6th European Particle Accelerator Conference, Stockholm, Sweden, 22 - 26 Jun 1998; Ed. by S. Myers, L. Liljeby, and C. Petit-Jean-Genaz Bristol, IOP, 1999. See also I. Mikulec, these proceedings.

2. I. Mikulec, these proceedings.

3. L.M. Sehgal and M. Wanninger, Phys. Rev. D 46 (1992) 1035; Erratum-ibid. D 46 (1992) 5209.

4. P. Heiliger and L.M. Sehgal, Phys. Rev. D 48 (1993) 4146; Erratum-ibid. D 60 (1999) 079902.

5. E.J. Ramberg et al., Phys. Rev. Lett. 70 (1993) 2525.

6. A. Alavi-Harati et al., Phys. Rev. Lett. 84 (2000) 408 .

7. K. Senyo, Proceedings of the International Europhysics Conference on High Energy Physics, Tampere, Finland, 15 - 21 Jul 1999; 
Ed. by K. Huitu, H. Kurki-Suonio and J. Maalampi - Bristol, IOP, 2000.

8. H. Bergauer et al., Nucl. Instr. and Methods A 419 (1998) 623.

9. A. Lai et al., to be published in Phys. Lett. B.

10. H.B. Greenlee, Phys. Rev. D 42 (1990) 3724.

11. G.D. Barr et al., Phys. Lett. B 351 (1995) 579 .

12. J.F. Donoghue, private communication.

13. A. Lai et al., to be published in Phys. Lett. B.

14. G. D'Ambrosio et al., JHEP 08 (1998) 004.

15. G.D. Barr et al., Phys. Lett. B 304 (1993) 381.

16. A. Zinchenko, these proceedings.

17. C. Geweniger et al., Phys. Lett. 57B (1975) 193.

18. A. Angelopoulos et al., Phys. Lett. B 425 (1998) 391.

19. M.N. Achasov et al., Phys. Lett. B 459 (1999) 674.

20. A. Apostolakis et al., Phys. Lett. B 456 (1999) 297.

21. Particle Data Group, Eur. Phys. J. C 15, (2000) 1.

22. The Second DA $\Phi$ NE Physics Handbook; Ed. by L. Maiani, G. Pancheri and N. Paver, INFN, LNF, 1995.

23. R. Batley et al., Addendum 2 to P253: A high sensitivity investigation of $K_{S}$ and neutral hyperon decays using a modified $K_{S}$ beam. CERN/SPSC/2000-002, 1999.

24. A. Alavi-Harati et al., Phys. Rev. Lett. 83 (1999) 22.

25. V. Fanti et al., Phys. Lett. B 465 (1999) 335.

26. R. Batley et al., Addendum 3 to P253: Precision measurement of charged kaon decay parameters with an extended NA48 setup. CERN/SPSC/2000-003, 1999.

27. A. Belkov et al., Phys. Lett. B 300 (1993) 283.

28. L. Maiani and N. Paver, The Second DA $\Phi$ NE Physics Handook, Vol. 1, p.51; Ed. by L. Maiani, G. Pancheri and N. Paver, INFN, LNF, 1995.

29. G. D'Ambrosio et al., Phys. Lett. B 480 (2000) 164.

30. W.T. Ford et al., Phys. Rev. Lett. 25 (1970)
1370.

31. L. Rosselet et al., Phys. Rev. D 15 (1977) 574.

32. S. Pislak, LNF seminar, 22 Jun 2000.

33. G. Barouch et al., Nucl. Instr. and Methods A 423 (1999) 32. 